\title{
Assessment of Iron Status in Cases of Anemia: A Comparative Analysis by Gale's and Intensive Method
}

\author{
Sanjay Kaushik, Neelima Bahal*, Sanjeev Kishore, Seema Acharya, Ishita Goel and Rajnish Kumar \\ Dept. of Pathology, Shri Guru Ram Rai Institute of Medical \& Health Sciences, Dehradun(India)
}

\begin{abstract}
Background: Nutritional anemia particularly iron deficiency anemia comprise a major public health problem in India. Precise diagnosis is necessary for the correct treatment as the multiple coexisting morbid chronic inflammatory conditions seen in these cases gives erroneous biochemical results. Microscopic assessment of bone marrow iron is considered to be the the gold standard. However, the conventional Gale's method cannot differentiate between the iron deficient cases and functional iron deficiency. This is made possible by the newer intensive method of iron assessment in the present study.
\end{abstract}

Aim: To compare the assessment of iron status by Gale's and Intensive method on bone marrow aspirate smears in cases of anemia.

Materials and Methods: A prospective study of 117 cases of all age groups and both sexes, having haemoglobin $<10 \mathrm{mg} / \mathrm{dl}$ were included in the study. Bone marrow aspiration was done and iron assessment was done by both the Gale's and Intensive method. Serum ferritin was also done in 23 out of 117 cases to serve as a control. However, it was not a part of the study. Results: Gale's method revealed hypoferrimic state in $29.1 \%$ cases and normal iron stores in $70.9 \%$ cases. Iron deficient status was further reduced to $6.83 \%$ cases by the intensive method of iron assessment. Maximum number of cases had functional iron deficiency by the intensive method.

\section{Keywords: Anemia, Bone Marrow Iron, Gale's Method, Intensive Method}

\section{Introduction}

Nutritional anaemia, particular iron deficiency anaemia is a major health problem worldwide especially in developing countries. ${ }^{[1]}$ The third national family health survey (NFHS -III) which was conducted in 2005-2006 estimated high prevalence of anaemia in India, with 79\% children, 70\% women and $24 \%$ adult men affected. ${ }^{[2]}$ A combination of markers, namely serum ferritin, serum iron, TIBC and percentage saturation of transferrin are being routinely used to assess iron stores of individual. ${ }^{[3,4,5,6]}$ Measurement of S. ferritin is currently the accepted non invasive test for assessing tissue iron stores. ${ }^{[4]}$ However, S. ferritin is an acute phase reactant which is raised in conditions associated with inflammation, liver disease, malignancy and hemodialysis. Therefore the use of S. iron markers may not discriminate between depleted iron stores and conditions associated with defective reticuloendothelial release of iron (functional iron deficiency). ${ }^{[4,7]}$ Microscopic examination of Prussian blue stained bone marrow has been considered the "gold standard" for determing iron depleted states. ${ }^{[7,8]}$ Conventionally, iron has been primarily assessed in marrow fragments which represent iron stores in the form of hemosiderin. Although some studies have shown a correlation between histological iron grading and chemical iron concentration in bone marrow, some have raised questions on the validity of histological grading. ${ }^{[9,10]}$
Keeping in view the importance of assessing iron status, we conducted this study to assess and classify the iron status in bone marrow by both Gale's and Intensive method. ${ }^{[7,9]}$

\section{Material and Method}

This prospective study was carried out in the department of Pathology, SGRRIM\&HS, Shri Mahant Indiresh Hospital, Dehradun over a period of two years (2013 -2015). A total of 117 cases of anaemia with haemoglobin less than $10 \mathrm{~g}$ / dl were included in the study. This study was vetted by the institutional ethical committee. Peripheral venous blood was collected for the assessment of haemoglobin and other haematological parameters. Bone marrow aspirate was obtained after written informed consent from posterior superior iliac spine under strict asepsis. Perl's Prussian blue stain using neutral red as a counterstain was used for studying the iron status. A positive control was included in each batch of slides. Marrow smears with at least seven fragments were considered adequate and were subjected for microscopic examination. All the marrow smears were then systematically assessed using Gale's and Intensive methods. The Gale's grading system was used to assess marrow fragments. Next, the intensive method was used to assess marrow iron in three sites i.e, fragments, macrophages around the fragments and erythroblasts. Twenty fields around the fragments were examined under oil immersion for the presence of macrophage iron and 100 
erythroblasts were examined. Iron status graded according to Gale's method was categorised from grade 0 to grade 6 (Table 1). Grade 0 and grade 1 corresponding to none and very slight marrow iron were considered indicative of iron deficiency. Categorisation of bone marrow iron status by intensive method was done into normal, functional iron deficiency, iron store deficiency and combined deficiency (Table 2). Since S. ferritin has been considered as a standard for assessing iron stores for so many years, it was done in 23 cases. This served as a control as well as helped in comparing the results of iron assessment with bone marrow.

\section{Result}

Of the total 117 cases, $49 \%(57 / 117)$ were males and $51 \%(60 / 117)$ were females. The minimum age was 4 years and maximum age was 93 years. 37.6\% (44/117) cases presented with mild anaemia $(8-10 \mathrm{~g} / \mathrm{dl}), 32.4 \%($ $38 / 117)$ cases presented with moderate anaemia $(6-8 \mathrm{~g} / \mathrm{dl})$ and severe anaemia $(<6 \mathrm{~g} / \mathrm{dl})$ was seen in $30 \%(35 / 117)$ cases. The minimum haemoglobin noted was $2 \mathrm{~g} / \mathrm{dl}$ and the maximum was $9.8 \mathrm{~g} / \mathrm{dl}$.In the present study, Gale's study revealed hypoferrimic state in only 34 cases out of $117(29 \%)$. According to intensive method, out of these 34 cases, only 8 cases were actually deficient cases that too had combined deficiency, 22 cases had functional iron deficiency and 4 cases had normal iron stores (Table 3). In the present study, according to the intensive method, maximum number of cases had functional iron deficiency (67/117). There were no pure iron deficient cases and 12 out 117 cases revealed combined deficiency (Table 3 ).

Table 1: Grading for bone marrow iron status according to Gale et al. (Figure 1)

\begin{tabular}{|c|c|c|}
\hline Grade 0 & None & No visible iron under high power magnification (x1000) \\
\hline Grade 1 & Very slight & Small iron particles just visible in few reticulum cells under high power magnification $(x 1000)$ \\
\hline Grade 2 & Slight & Small, sparsely distributed iron particles just visible under low power magnification (x100) \\
\hline Grade 3 & Moderate & Numerous small iron particles in reticulum cells throughout the marrow fragment $(x 100)$ \\
\hline Grade 4 & Moderate heavy & Larger iron particles throughout the fragment with tendency to aggregate into clumps $(x 100)$ \\
\hline Grade 5 & Heavy & Dense, large clumps of iron throughout the fragment $(x 100)$ \\
\hline Grade 6 & Very heavy & $\begin{array}{l}\text { Very large deposits of iron, both intra and extra cellular, obscuring cellular detail in the } \\
\text { fragment }(x 100)\end{array}$ \\
\hline
\end{tabular}

Table 2: Classification of iron status using the Intensive grading method. (Figure 2).

\begin{tabular}{|l|l|l|l|}
\hline Fragment & Macrophage & Erythroblast & Iron status category \\
\hline Present & Present & Present & Normal \\
\hline Present & - & Present & Normal \\
\hline Present & Present & - & Functional iron deficiency \\
\hline Present & - & - & Functional iron deficiency \\
\hline- & Present & Present & Iron stores deficiency \\
\hline- & - & Present & Iron stores deficiency \\
\hline- & Present & - & Functional and iron stores deficiency \\
\hline- & - & - & Functional and iron stores deficiency \\
\hline
\end{tabular}

Table 3: Comparison of Grades of both Gale's and Intensive method of the all the cases included in the study.

\begin{tabular}{|l|c|c|c|c|c|}
\hline \multirow{2}{*}{ Gale's Method } & \multicolumn{5}{|c|}{ Intensive Method } \\
\cline { 2 - 6 } & Normal & Functional Iron Deficiency & Iron store Deficiency & Combined Deficiency \\
\hline Grade 0 & 02 & 00 & 00 & 08 & $\mathbf{1 0}$ \\
\hline Grade 1 & 02 & 22 & 00 & 00 & $\mathbf{2 4}$ \\
\hline Grade 2 & 04 & 22 & 00 & 02 & $\mathbf{2 8}$ \\
\hline Grade 3 & 17 & 03 & 00 & 00 & $\mathbf{2 2}$ \\
\hline Grade 4 & 06 & 08 & 00 & 00 & $\mathbf{1 4}$ \\
\hline Grade 5 & 05 & 08 & 00 & 00 & $\mathbf{1 3}$ \\
\hline Grade 6 & 02 & 04 & 00 & $\mathbf{1 2}$ & $\mathbf{1 1 7}$ \\
\hline Total & $\mathbf{3 8}$ & $\mathbf{6 7}$ & $\mathbf{0 0}$ & & 02 \\
\hline
\end{tabular}



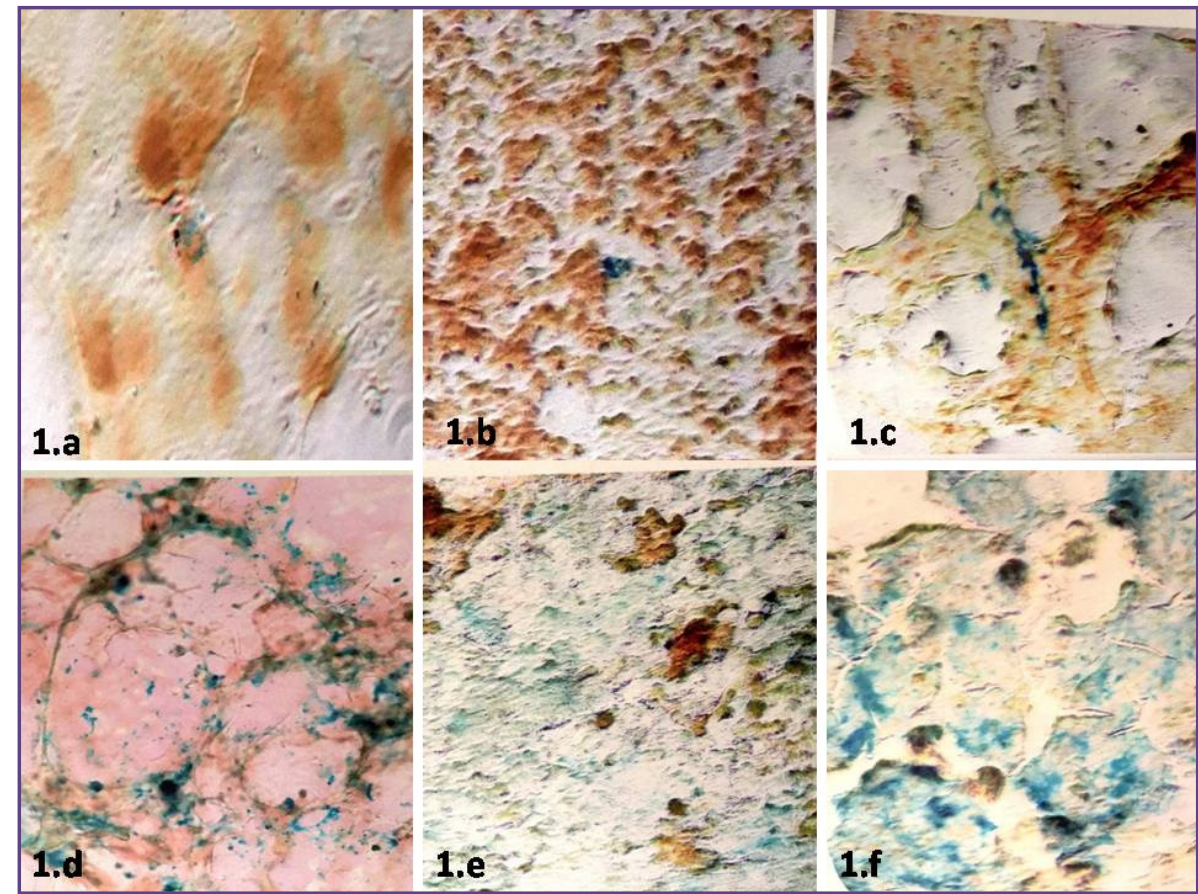

Fig.1: bone marrow smear showing a) small iron particles just visible under high power magnification Gale's Grading-1; b) small sparsely distributed iron particles in marrow fragment- Gale's Grading-2; c) numerus small iron particles in marrow fragment- Gale's Grading-3; d) large iron particles in throughout marrow fragment with tendency to aggregates in clumps Gale's Grading-4; e) dense large clumps of iron in throughout marrow fragment - Gale's Grading-5; f) very large deposits of iron particles both in intra- \& extra-cellular marrow fragment - Gale's Grading-6 (Perl's Prussian Blue stain. 1000x \& 400x).

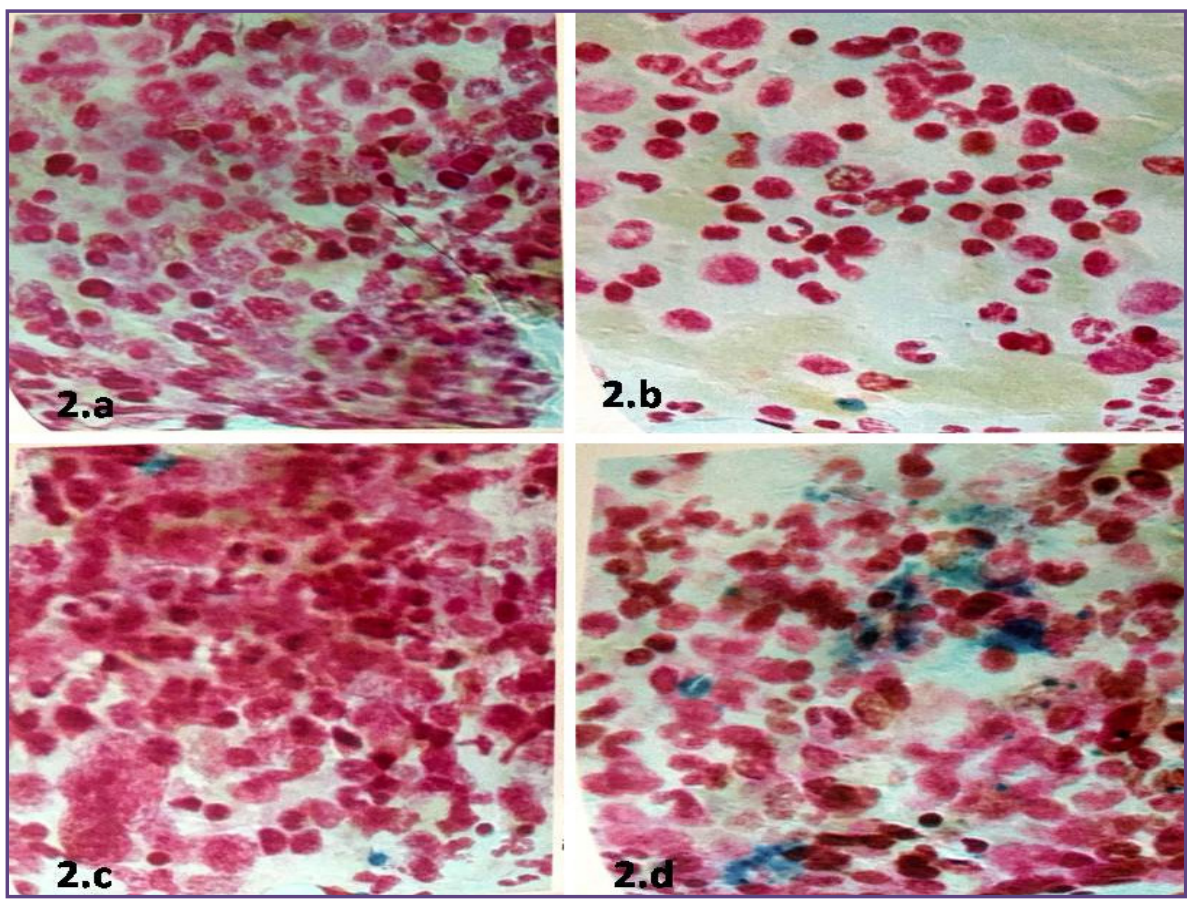

Fig. 2: Bone marrow smear showing a) macrophages \& erythroblasts in the marrow fragment with no visible iron (Functional \& iron store deficiency) b) iron in both marrow fragment and macrophages (functional iron deficiency); c) iron only in the marrow fragment (functional iron deficiency); d) iron in marrow fragments, macrophages \& erythroblasts (Normal iron) (Perl's Prussian Blue stain counter stained by Neutral Red. 1000x) 


\section{Discussion}

As is well known, that iron deficiency anaemia is a nutritional anaemia. But functional iron deficient cases constitute a large percentage of these cases and they need to be segregated from the pure iron deficient cases. Functional iron deficiency develops when normal physiological system for transporting iron to target tissues is impaired inspite of the presence of satisfactory iron stores. ${ }^{[12]}$ This occurs due to the release of cytokines as an acute phase response to infection, leading to impaired erythropoiesis and anaemia which is also known as 'anaemia of inflammation'. ${ }^{[13]}$ The clinical distinction between iron deficiency anaemia and functional iron deficiency is important to avoid unnecessary iron supplementation. ${ }^{[14]}$ In this study, all the patients were anaemic and were being prescribed iron on an empirical basis without proper assessment of their iron status. Later on, it was found that the actual number of patients requiring iron was very low and most of the patients were being wrongly treated. It is very important to diagnose the iron deficient cases, before prescribing iron and moreover wrong and overtreatment can lead to toxicities and the approach to correct diagnosis is diverted.The literature shows that the various studies in the past have included S. ferritin and other markers as an important component for diagnosing iron deficiency. It reflects the total body iron stores and a low level indicates hypoferremic state. ${ }^{[3,6]}$ On the contrary, it is well known that low S. ferritin can diagnose iron deficiency but high $\mathrm{S}$. ferritin does not necessarily exclude iron deficiency. It is a false indicator for iron deficiency in chronic inflammatory cases which constitute a major percentage of anaemic cases. It also gives a false sense of security to the iron deficient cases. It has already been proven in the present study that functional deficient cases comprise maximum percentage of anaemic cases. There were $57.26 \%$ (67/117) functional deficient cases by the intensive method of iron assessment in the present study. This was also seen in another study by Phiri et al, in which the functional iron deficiency was the most common iron status category comprising 39.6\%(74/187) cases. ${ }^{[7]}$ In all these cases, S. ferritin would be raised and cannot be used as a marker of iron status. Considering this fact, S. ferritin was excluded from the present study. This study demonstrated iron deficiency in $29.1 \%$ cases and normal iron status in $70.9 \%$ cases, which is correlating with the results of another study by Bableshwar et al. ${ }^{[15]}$ They found iron deficiency in $38.75 \%$ cases and normal iron stores in $61.25 \%$ cases. These $29.1 \%$ cases $(34 / 117)$ were further narrowed down to $6.83 \%(8 / 117)$ cases by the intensive method. Hence, the results of the present study prove the superiority of the intensive method for assessing iron over the Gale's method. Studies have shown the frequent use of Gale's method for assessing iron by bone marrow but now the intensive method is proved to be better for diagnosis and specificity. It segregates the iron deficient cases from functional deficient cases which is a drawback in Gale's method of iron assessment. Intensive method can accurately diagnose the iron deficient cases and helps in correct treatment. It would not be wrong to accept the difficult technique and the expertise that is required for the bone marrow aspiration. Studies have even criticised the use of bone marrow for iron assessment because it is an invasive method and is considered subjective. ${ }^{[12]}$ The importance of bone marrow iron staining cannot and should not be ignored in the gray zone conditions where the use of $\mathrm{S}$. iron markers gives borderline and fallacious results. Keeping in view this criticism, it is advisable to do the $\mathrm{S}$. iron studies in all anaemic cases. Cases having raised S. ferritin should be segregated especially if the history suggests the association of any inflammatory condition. These cases should be subjected to iron assessment by bone marrow examination for getting precise results.

\section{Conclusion}

Differentiation between iron deficiency anaemia and functional iron deficiency is important for the correct treatment of the patient. S. iron parameters should be used for the diagnosis of iron deficiency anaemia, but our observation emphasise the use of bone marrow iron staining as a valuable test for excluding iron deficiency anaemia in patients with indeterminate biochemical indices. Although intensive method of grading iron requires more expertise, it has proved to be superior and more precise in diagnosing iron deficient cases.

\section{References}

1. Looker AC, Dallman PR, Carroll MD, Gunter EW, Johnson CL. Prevelance of iron deficiency in the United States. JAMA 1997: 277; 973-6.

2. NFHS India 2005-06, National Family Health Survey-III (NFHS -III). Anemia among women and children, Mumbai: International institute for population sciences 2006.

3. WHO. S. ferritin concentration for the assessment of iron status and iron deficiency in population. Vitamin and mineral nutrition information system. Geneva: World Health organisation; 2011.

4. Krause JR, Stole VS. Ferritin and bone marrow iron stores and correlation with absence of iron in biopsy specimens. Am J Clin Pathol 1979; 72: 817-20.

5. Baynes RD. Assessment of iron status. Clin Biochem. 1996; 29: 209-15

6. Hughes DA, Stuart - Smith SE, Bain BJ. How should stainable iron in bone marrow films be assessed? J Clin Pathol 2004; 57: 1038 -40. 
7. Phiri KS, Calis JC, Kachala D, Borgstein E, Waluza J, Bates I et al. Improved method of assessing iron stores in bone marrow. J Clin Pathol 2009;6: 685-9.

8. Stuart-Smith SE, Hughes DA, Bain BJ. Are routine iron stains on bone marrow trephine biopsy specimen necessary? J. Clin Pathol 2005; 58: 269-72.

9. Gale E, Torrence J, Bothwell T. The quantitative estimation of total iron stores in human bone marrow. J Clin invest 1963; 42: 1076-82.

10. Johansson SV, Plantin LO, Strandberg PO, et al. Estimation of iron in human bone marrow with histological, chemical and neutron activation analysis. Clin Chem Acta 1970; 30: 549-58

11. Ebru K, Deniz A C, Yahya B, et al. Bone Marrow iron staining is a reliable test for elimination of iron deficiency anemia rather than its diagnosis. International $\mathrm{J}$ of Hematol and Oncology 2013;4: 23:260-3.

12. Roy CN, Andrews NC. Anemia of inflammation: hepcidin link. Curr Opin Hematol. 2005;12: 107-11.

13. Trey JE, Kushner I. The acute phase response and the hematopoietic system: the role of cytokines. Crit Rev Oncol Hematol 1995; 21: 1-18.

14. Wish JB. Assessing iron status: Beyond serum ferrtin and transferrin saturation. Clin J Am Soc Nephrol 2006; 1: 84-8.

15. Bableshwar RS, Roy M, Bali A, Patil V P, Inumella S. Intensive method of assessment and classification of bone marrow iron status: A study of 80 patients. Indian J of Pathol and Microbiol. 2013; 56(1) : 16-19.

*Corresponding author:

Dr. Neelima Bahal, Assistant Professor, Dept of Pathology, Shri Guru Ram Rai Institute of Medical \& Health Sciences, Dehradun-248121 (India)

Phone: +919411401101

Email: drneelimasuri@gmail.com

Financial or other Competing Interests: None. 